\title{
An Anticipative Effects-Driven Approach for Analyzing Interoperability in Collaborative Processes
}

\author{
Nicolas Daclin and Vincent Chapurlat \\ LGI2P - Laboratoire de Génie Informatique et d'Ingénierie de la Production \\ Site EERIE de l'Ecole des Mines d'Alès, Parc Scientifique G. Besse \\ 30035 Nîmes cedex, France \\ \{nicolas.daclin, vincent. chapurlat\} @ema.fr
}

\begin{abstract}
Partners involved into a collaborative process must satisfy interoperability requirements in order to fulfil adequately their mission all along the process. Indeed, interoperability is now considered as a key factor of success when sharing data, services, knowledge, skills, and resources. However, although the main desired effects (to respect a cost, to produce $\mathrm{n}$ products, to respect a quality ratio...) of this process are generally reached, some others effects (unpredictable, undesirable...) may be also induced and can lead in some cases, to a worsening of these desired effects that process has to perform. This paper presents and illustrates an Anticipative Effects-Driven Approach to check interoperability rules in collaborative process model.
\end{abstract}

Keywords: Interoperability, anticipative effects-driven approach, collaborative processes.

\section{Introduction}

Numerous initiatives $[1 ; 2]$, developed from the last years and dealing with enterprises interoperability, have shown that ability for several partners to be interoperable is a key factor of the partnership's success. Moreover, considered for a long time only as a problem of computer sciences [3], these initiatives have demonstrated that enterprises interoperability is now considered as crucial in enterprise [4], relevant in research area [5] and can take place at different levels [6] (e.g. business, process, services...). In the limited frame of interoperability in process, partners are interoperable if they are able to share data, services, skills, resources. Thus, developing interoperability become crucial in order to ensure the success of the whole process and therefore to ensure a given level of performance, efficiency, reactivity and agility of this process. The waited effect of a considered process is to fulfil a given mission (to develop a new product, to find solutions facing a customer problem...) and to reach performance objectives in terms of costs, duration, and quality of service/product (i.e. adequacy between product/service, partners' needs and customer demand). However, this process can be affected by a lack of interoperability from a partner with others during the process runtime. As a consequence, allowing partners to know their potential to interoperate prior to the execution 
could help (1) partners to anticipate a worsening of the achievement of process's objectives and thus (2) to adapt the process as far as possible. In this case it is about to analyze each effect that a partner can induce on the process in terms of interoperability. The purpose of this communication is to present the principles and to illustrate an Anticipative Effect-Driven Approach that allows partners to build an appropriate process and then to better control this one in the context of collaboration.

\section{Interoperability Analysis}

Generally speaking, interoperability represents the ability for two or more systems to operate together (or in conjunction). In the context of networked enterprise, interoperability takes place at various levels of the enterprise and has to consider various barriers as presented in the interoperability framework given in [7]. This one allows identifying and structuring the different fields in which interoperability must be analysed and improved. The goal is to develop relevant knowledge and solutions to remove, at least, one interoperability barrier at one enterprise level. Thus, this paper focuses on the analysis of interoperability in collaborative processes [8]. It is defined as the capability and the capacity of numerous resources, flows, processes, organization units (i.e. teams, departments) in enterprises, to work together with limited risks and without lost of performance, integrity, stability and autonomy. In this context, interoperability analysis has to be seen under two hypotheses: (1) Interoperability concerns each partner considered separately. The interest, in this case, is to know if this pointed out partner is able (or not, or in some measure yet) to perform a required service in time, a required ability at the right place and so on independently from other partners of the collaborative process. (2) Interoperability concerns all the possible interactions, their nature and dynamic all along the process runtime. The analysis of interoperability must focus first on interactions between activities of the process and second between partners themselves. Activities have to be synchronized and controlled, including the structure of the process (parallelism, sequence, mutual exclusion between activities...). As a consequence, interoperability - precisely interoperability in processes - can be seen as a set of requirements that partners will have to respect. Thus, this research work focuses on the questions: "How is it possible to define interoperability problematic? How is it possible to detect when and how some possible worsening effects due to lack of interoperability can occur? How is it possible to anticipate them?". The objective is to bring to partners, involved in the process execution, the knowledge about the potential effects that they can generate. In this case, partners will have the possibility either to validate the process and to start its execution or to improve it by providing alternatives. To do that, interoperability requirements have to be conceptualized and analyzing rules have to be implemented in order to detect if these requirements are fulfilled to avoid interoperability problems. Moreover, ensuring interoperability in process implies to define a modeling language and tool or to enrich existing ones according to interoperability requirements concept. Several modeling tool are effectively available but none allow an analysis of the possible effects and especially effects due to a lack of interoperability. 


\section{AEDA Principles}

The proposed approach, named Anticipative Effect-Driven Approach (AEDA), allows to model, to analyse and to assess the interoperability according to the two hypotheses presented above. It is based on existing approaches developed in other fields of application (military, civil crisis response...). With regard to the approaches that take an interest on the assessment of effects that a system can produce before its implementation, the Failure Mode and Effects Analysis (FMEA) [9] is the most widely spread and used. Some extensions of this method, such as Process-FMEA, have been developed since. In this case, the objectives of the P-FMEA is to establish a set of "potential failure modes" and "effects of failure" that can occur during the process runtime in order to take corrective action before the process implementation. However this kind of method is essentially based on a brainstorming procedure and applied every time that a process has to be implemented. Others approach such as the Effect-Based Operation [10] consists to characterize and to evaluate the possible effects of actions which are supposed to reach a final outcome. In opposition to the FMEA, EBO is not based on the research of potential failure and effects that an action in a process can generate, but on the research of the outcome that actions have to achieve. In the case of collaborative processes facing to interoperability problems, these approaches remain interesting in their principles that consist to observe and to analyse the effects resulting from the execution of the process. They must be however enriched and conceptually rethought in order to match with the requirements presented before i.e. to characterize from a generic manner, to detect and above all to anticipate interoperability problems. As a consequence, the AEDA approach has to provide: (1) A set of modeling concepts and relations between concepts allowing partners to describe their characteristics and their role in the collaborative process in order to become able to reply to the questions such as: "Am I able to perform effectively and efficiently a task? and, if a failure is highlighted, in which measure this one can affects the entire collaborative process?". (2) A set of (as possible) generic interoperability requirements that have to be respected all along a collaborative process. The goal is to help partners to analyze their role, capacities and abilities within the process. (3) A mechanism for characterizing and detecting on the process model what, where and how can be the potential effects induced when a problem of interoperability occurs.

\subsection{Modeling Concepts}

As mentioned before, several concepts are required to implement the approach. The first ones are related to the process domain and coming from systemic domain, system engineering domain, enterprise modelling domain. They are justified to model properly the different configurations and characteristics of the collaborative process. Basically, processes are sequences of activities modelled from their start to their end. Activities transform and input state into an output state, use a set of resources (human and/or material as well) and are controlled. Thus, to make sure that an activity will be perform in a good way, some knowledge about the triplet resource, control and the activity itself are needed to be collected and implemented. To do that, the frame of reference time space and shape [11] can allow this collect about any elements involved in the process. Indeed, by the adaptation of this frame it is possible to position 
an activity, a resource and a control in this frame. This knowledge, represented in the form of attributes that characterize the considered element can be independent of any domain of application - e.g. resource capacity, availability, pre-emption... [12] - or specific to domain e.g. an activity can required a certain level of protection from its resources...It is based on this enrichment, that interoperability requirements can be developed and allow, thereafter, to reason about the possible effects about their achievement or not.

\subsection{Interoperability Requirements Modelling}

The others concepts required in the AEDA approach are related to (1) the design of interoperability requirement and (2) the effect characterization. The first one has to allow defining all the requirement a partner has to respect in the process. The second one enables to evaluate in which way the collaborative process may be affected if this set of requirements if not satisfied. According to the property concept proposed by [13], each interoperability requirement is modelled by a set of properties. A property is a causal temporized and constrained relation between two predicates called respectively cause or Condition and effect or Conclusion. Cause and effect are described by using variables or attributes and predicates or functions extracted from the collaborative process model. These variables characterize each element involved into the process (resource, flow, activity, etc.), taking into consideration the frame of reference time shape and space (TSS). It allows defining and formalizing physical attributes which characterizes any element, from a quantitative or qualitative manner, evolving in the time, in the space or in its shape. Any element may be "a part of" or "interacts" with another element. In this case, the evolution of each element affects and modifies the referential of the surrounding elements. Thus, defining which elements evolve in a given referential allows to know the impact of these elements on their environment. In order to refine the characterization of an element in terms of time shape and space, these ones are decomposed into sub attributes. The time attribute is defined by the sub-attributes called date and duration. The space attribute is defined by the subattribute called location in a defined space. The shape attribute is defined by the subattributes such as capacity, dimension, vulnerability (improvement or degradation of the object), quantity, etc.. The causal relation describes the nature of the link between the condition and the conclusion (implication, equivalence or influence) and details what are the temporal constraints on which this relation is established. An example of property is illustrated in natural language and by using TSS and predicates in table 1.

Table 1. Example of property

\begin{tabular}{lll}
\hline Cause & Relation & Effect (conclusion) \\
\hline $\begin{array}{l}\text { Exists a in Activities, forall } \mathrm{x} \text { in Partner, [re- } \\
\text { quiredAptitudes(a) in aptitudes(x) and location(x) }\end{array}$ & $\Rightarrow$ & {$[\mathrm{x}$ in elligibleResourcesOf } \\
$=$ location $(\mathrm{x})$ and availability $\mathrm{t}]$ & $\mathrm{(a)}=\mathrm{a})$ \\
\hline
\end{tabular}

The set of property provides a support of reasoning allowing verifying the collaborative process model in respect of the interoperability requirements. However, if a given property is not satisfied by the model, it is also necessary to evaluate finely the 
real impact of this lack i.e. to characterize what can be the resulting effect on the process runtime.

\subsection{Effect Characterization}

An effect can be defined as a situation that can be expected, undesired, dreaded and results from an interaction. An effect results always and only from an interaction between one object, defined as the source, and one or several objects, defined as the destination. It can be simply modeled by the possible variation (or dependence) between one or several TSS attributes of the destination under the action of the source. An effect can be (1) predictable i.e. assessable and observable indicators exist either on the source object or the destination object(s); (2) potential i.e. a logic relationship between the cause and the effect exist and; (3) unpredictable or emergent, this kind of effect is not taken into consideration by the approach. An effect can be defined as direct or indirect taking into account the causal relation between situations which have induced the effect. The goal is now to characterize the nature of an effect by determining if it is [14]: Harmful, this effect is produced when the source induces a deterioration of the characteristics of the destination. These kinds of effects have to be annihilated. Good, this effect is produced when the source induces a variation of the characteristics of the destination as expected. These kinds of effects have to be maintained. Excessive, this effect is produced when the source induces a variation of the characteristics of the destination beyond this expected. In this case the effect has to be reduced. Insufficient, this effect is produced when the source induces a variation of the characteristics of the destination less than expected. The effect must be improved in order to become efficient. Furthermore, an effect is then modelled as property. For example, the property defined as: Forall a in Activities, Forall element in input(a) [requestTSSInput(a) in TSS(element)] $\Rightarrow[\operatorname{effect}(a$, element $):=\operatorname{good}]$ means that if the TSS attributes requested by a given activity in input contains the TSS attributes of the element considered to be processed then the effect of the activity on the element is considered as good and this for all the activities of the collaborative process. This property can be decomposed into sub properties specifying the way to interpret the variation of effect value taking into account more precisely a given subset of TSS attributes of the activity and/or of the element. Thus, the following property: Forall $a$ in Activities, forall aptitude in requestedAptitude(a), forall partner in elligibleResourcesOf (a) [requestedCapacity(aptitude,a) < capacity (partner)] $\Rightarrow$ [effect (partner, a):= insufficient and elligibleResourcesOf $(a):=$ elligibleResourcesOf (a)-partner] means that if an activity requires precisely a given capacity (evaluation i.e. quantification or qualification of a given aptitude taking into account a common scale of measure) then each partner selected as a potential resource must provide this capacity in order to be qualified, otherwise the effect of t element has a sub attribute quantity less than to the sub-attribute quantity describing the expected quantity of element by the activity i.e. the quantity of inputs from this partner on a is considered as insufficient.

\section{AEDA in Use}

All the concepts related the characterization of (1) the elements involved in the process and (2) effects resulting from its execution are synthesized in Figure 1. 


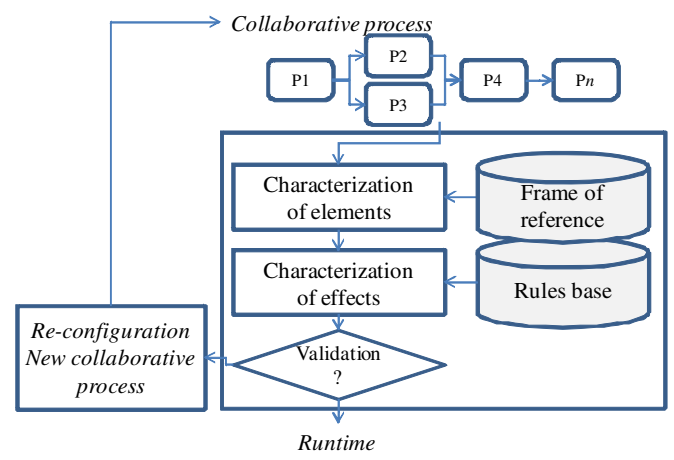

Fig. 1. The structure of the Anticipative Effects-Driven Approach

The first step consists to characterize the elements involved in the collaborative process. In this phase, the objective is to collect a maximum of information about the different activities, the resources that have to perform activities and controls. This characterization is based on the use of the TSS concept: each attribute of each element is detailed. Then the nature of the potential effects (harmful, good, insufficient, excessive and absent) induced by the collaborative process has to be determined. This step is performed thanks to the referential of interoperability requirements and effect characterization properties. At the end of this step the collaborative process is either validated or rejected. If the process is approved, the partners validate its planning and/or perform some adjustments and start its execution. If the process is rejected, this one can be reconfigured or fully redeveloped. The new configuration is one more time submitted to the approach in order to detect other effects. Currently, the approach is developed in the French research project, dealing specifically with the interoperability of systems in crisis situation, ISYCRI (Interoperability of Systems in CRIsis situation, ANR-06CSOSG). In order to illustrate the interest of the here presented approach, a specific collaborative process coming from a case study (NRBC exercise managed by the Prefecture of the Tarn in France, $27^{\text {th }}$ February 2004) is used [15]. This scenario starts with the description of the crisis situation. The scenario which has been chosen is the following: "At 10 AM, on 27th February 2004, the police is informed that an accident among a tanker truck (unknown substance) and a wagon containing chemical products is occurred. The policemen are sent on the scene and the employees of the railway station fall unconscious while several children of the neighboring kindergarten feel sick". A first collaborative process is created in order to face up the crisis. It consists in the execution of different activities both in parallel and in sequence. The execution of each activity required a given actor (or resource). Thus, the objective of the anticipative effect-driven approach is to validate the given collaborative process before its execution. Figure 2 shows the collaborative process that has to be implemented.

The first step is to collect, as far as possible, a maximum of knowledge related to element involved in the process i.e. about the different activities and the resource requested to perform them. Then, based on this knowledge collected, the potential effects that could occur in the process have o be determined. Let consider the interaction between the human resource "Red Cross" that have to perform the service "set medical post". If set medical post required an end date, it implies that the resource 


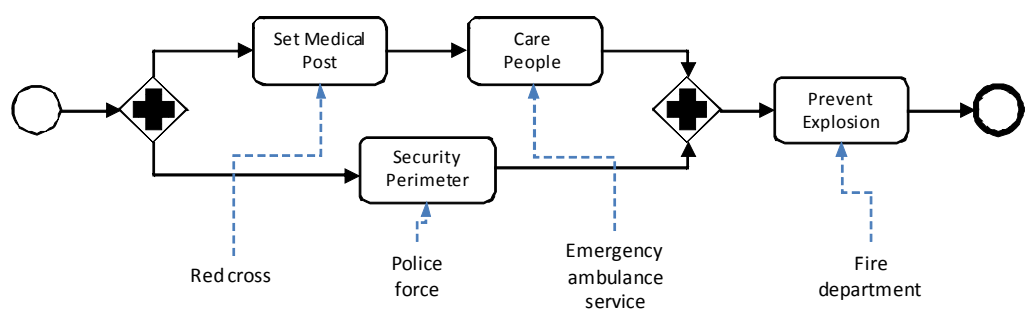

Fig. 2. Example of NRBC crisis management process

over the service at the required end date. If this requirement is effectively verified, then the effect of Red Cross on the service can be characterized as good. Conversely, if the requirement is not verified, it is question to know in which measure this requirement is violated. Does the end date will be superior or less than the expected end date? In the first case the effect will be characterize as insufficient in the second, as excessive. Then what is the effect on the first activity on the next one (care people), does its execution will be delayed or not? Thus the anticipative effect driven approach try to evaluate this set of effect all along the collaborative process and prior to its execution. Obviously, a complete process analysis has to be performed in order to characterize each effect and to provide a maximum of information to the partners. In order to do that in an automatic way, an application is currently developed ("E.D.A. for Process Analysis". under Eclipse Graphical Modeling Framework) allowing (1) to model the collaborative process and (2) to model and to check the effects characterization between elements.

\section{Conclusion}

This paper presents an approach to help partner to increase their organizational interoperability at process level. Currently, the anticipative effect-driven approach is developed specifically in the context of crisis management. This one has to allow an adaptation and a validation of collaborative process by anticipating potential effects that could occur during the runtime phase. This adaptation and this validation has to lead to an improvement of the interaction between objects involved in the crisis in order to perform a collaborative process perfectly adapted to cope to the crisis. The concepts of the approach are clearly identified and a referential of property is currently developed in order to characterize the nature of the effects. Future work is concerned by the development of analyses rules that have to propose alternatives to managers and the development of the resolution algorithm that has to allow the building of new collaborative process.

\section{References}

1. Interoperability Research for Networked Enterprises Applications and Software (INTEROP Network of Excellence). Annex 1 - Description of Work (2003)

2. Advanced Technologies for Interoperability of Heterogeneous Enterprise Networks and their Applications (ATHENA). Integrated Project Proposal - Description of work (2003) 
3. IEEE. A compilation of IEEE standard computer glossaries. Standard computer dictionary (1990)

4. Chen, D., Dassisti, M.: Elveaeter. Enterprise Interoperability Framework and knowledge corpus - Final report. Interop deliverable Domain Interoperability, DI.3 (2007)

5. European Community Report (ECR) Unleashing the Potential of the European Knowledge Economy, Value Proposition for Enterprise Interoperability, Final Version (Version 4.0) (2008)

6. Enterprise Interoperability Research Roadmap (EIRR), Version 4.0, Cabral, R., Doumeingts, G., Li, M.S., Popplewell, K. (eds.) (July 31, 2006)

7. Chen, D., Daclin, N.: Framework for enterprise interoperability. In: 2nd IFAC Workshop on Enterprise Integration, Interoperability and Networking (EI2N), Bordeaux, France, March 20-24 (2006)

8. Chapurlat, V., Vallespir, B., Pingaud, H.: An approach for evaluating enterprise organizational interoperability based on enterprise model checking techniques. In: Proceedings of the 17th IFAC World Congress 2008, Seoul, Korea (2008)

9. IEC 60812. Analysis techniques for system reliability - Procedure for failure model and effects analysis (FMEA). Edition 2.0 (2006)

10. Lowe, D., Ng, S.: Effects-based operations: language, meaning and the effects-based approach. In: Proceedings of Command and Control Research and Technology SymposiumThe power of information age and technologies, San Diego, USA (2004)

11. Le Moigne, J.-L.: La théorie du système générale - théorie de la modélisation. Presse Universitaire de France (1977) (in French)

12. Vernadat, F.B.: Enterprise Integration Modeling and Integration: Principles and Applications. Capman \& Hall, London (1996)

13. Chapurlat, V., Kamsu-Foguem, B., Prunet, F.: A formal verification framework and associated tools for Enterprise Modeling: Application to UEML. Computers in Industry 57, 153-166 (2005)

14. Mann, D.: Hands on systematic innovation. CREAX Press Editor (2002)

15. Truptil, S., Bénaben, F., Couget, P., Lauras, M., Chapurlat, V., Pingaud, H.: Interoperability of Information in crisis management: crisis modeling and metamodeling. In: Proceedings of the 4th International Conference on Interoperability for Enterprise Software and Applications (I-ESA 2008), Berlin, Germany (2008) 